\title{
How Socio-Technical Factors Can Undermine Expectations of Human-Robot Cooperation in Hospitals
}

\author{
Kristina TORNBJERG ${ }^{\text {a, }}$, and Anne Marie KANSTRUP ${ }^{\text {a }}$ \\ ${ }^{a}$ Department of Planning, Aalborg University, Denmark
}

\begin{abstract}
This research analysed human-robot cooperation and interaction in the basement of a Danish hospital, where kitchen staff and porters conducted their daily routines in an environment shared with mobile service robots. The robots were installed to ease the everyday routines of kitchen staff and carry out physically demanding tasks, such as transporting heavy cargo between destinations in the hospital basement. The cooperation and interaction were studied through ethnographic inspired fieldwork and the results highlighted how robots affect the real-life environments into which they are gradually moving. The analysis revealed how the great human expectations of robots clashed with reality and identified three key elements that influence human-robot cooperation in hospitals: 1) environmental factors, 2) behavioural factors and 3) factors related to human reliance on robots. We emphasise the importance of considering socio-technical factors when deploying robots to cooperate with humans in hospital environments.
\end{abstract}

Keywords. Socio-technical factors, expectations of mobile robots in practice, hospital, human-robot cooperation

\section{Introduction}

Robots have gradually moved out of controlled settings, such as laboratories and industry, into everyday real-life environments, including homes, workplaces, cultural institutions and public arenas - not least hospitals, which is the setting with which this paper is concerned. As robots increasingly enter new application areas - 'the wild' - with diverse actors and unexpected responses, research on the relationship between humans and robots in real-life settings become more vital. Hence, knowledge about human reactions and behaviour towards robots in complex environments, and the influence of robots on the environment with which they interact, is essential. Robots are entering diverse social arenas, while most of the existing research within the field of human-robot interaction, which forms the basic understanding of how people interact and engage with robot technology, is concerned with controlled robot settings such as laboratories and industrial environments. There is a lack of research investigating how robots affect the uncontrolled environments in which they are deployed and therefore a lack of insights into what to expect when humans and robots cooperate in complicated environments and real-life work settings, such as hospitals. As a consequence, hospital staff tend to have greater expectations of robots than the robots are able to meet in practice. Hence, understanding

${ }^{1}$ Kristina Tornbjerg, Department of Planning, Aalborg University, Rendsburggade 14, 9000 Aalborg; E-mail: kristinat@plan.aau.dk 
the nuances and complexities of how humans and robots interact in hospitals (environments that are not highly structured, controlled or designed for robots originally developed to engage in industrial settings) is valuable for designing future human-robot cooperation-friendly hospital environments focused on socio-technical factors.

This paper reports findings from an ethnographic inspired study of hospital staff cooperating with mobile service robots in a hospital basement. The paper shows how challenges occur in human-robot teamwork in a hospital environment that is not designed for mobile robots, highlighting the socio-technical factors relevant to the robots' impact on the context of which they are a part. Mobile service robots were installed in the hospital basement to improve the physical wellbeing of a specific group of human service workers - hospital kitchen staff-by carrying out demanding physical tasks, such as transporting heavy cargo between destinations in the hospital basement.

\section{Background}

Related work within the field (mobile robots among humans in everyday environments) has researched how mobile robots affect the environments in which they are deployed. It has been known for some time that robots can change routines, affect activities and influence responsibilities; for example, Forlizzi researched the social impacts of mobile robots in homes and found that robotic vacuum cleaners could change peoples' cleaning activities and routines, influencing who held the responsibility for a household's cleaning tasks. In addition, the robot affected the nature of these tasks - not least the home ecosystem - as the social and cultural context of the home was modified by the presence and assistance of the robot [1]. Robots affect and impact the environment of which they are a part, which was also demonstrated in early work by Sung et al., who argued that domestic mobile robots could change household routines and established how technoenthusiasts tended to assign names, identities or/and personalities to their robots, leading to acceptance of domestic robots and emotional attachment to the technology [2].

When concentrating on mobile robots in work settings it becomes clear that a number of researchers have attempted to better understand the consequences of deploying mobile robots in dynamic work environments. Their research has shown how human perceptions of robots differ on account of the working environment and how the composition of those environments influence how humans use and experience robots. Early research by Mutlu and Forlizzi (2008) examined how different staff groups within a hospital perceived the same mobile service robot differently depending on different levels of acceptance of disturbances within their working environments across hospital wards [3]. Ljungblad et al. researched the reactions of hospital staff towards a mobile service robot in a hospital and proposed four different perspectives that staff might take in perceiving a robot, respectively an alien, a machine, a worker and a work partner. These perspectives can change over time, such as if a person develops a closer working relationship with the robot [4]. The recognition that robots affect the environments in which they are deployed laid the groundwork for our research, which investigated how robots impact the environment and what to expect when they are released into the wild. 


\section{Methods}

This study researched human-robot interaction and cooperation in a real-life work setting - a medium-sized hospital in Denmark - through an ethnographic inspired field study. In 2016, the hospital installed MiR Hook $100^{\mathrm{TM}}$ mobile service robots to relieve the hospital kitchen staff of the physically demanding task of transporting large carts with cutlery through the hospital basement. The basement is characterised as an unstructured real-life environment with mixed traffic made up of patients, clinicians, laboratory technicians, kitchen staff, porters, technical staff and workmen.

The ethnographic inspired field study, carried out in May 2020, consisted of 26 interviews with hospital employees (16 of whom were kitchen staff) recruited through snowball sampling with the aim of exploring their everyday practices and perceptions of working with the mobile service robots [5]. The study also included observations of two mobile robots performing their daily routines and tasks, operating without-and cooperating with - humans in the basement [6]. Finally, the kitchen staff took the First Author on guided tours during which they described their tasks and routines and explained how the robots were, or were not, involved in these [7][8]. The aim of mixing methods for data collection was to explore the setting; gain understanding of robots in the hospital; investigate the cooperation between this type of technology and humans; and to explore if human expectations towards cooperating with robots were concise, considering the robots' impact on the work setting.

The empirical data was in the form of descriptive notes, photos, video clips and audio files (equal to 10 hours of audio). The audio was transcribed and analysed (according to Braun and Clarke's techniques for thematic analysis [9]), along with the photographs, video and field notes. The empirical data was coded using themes identified in the data, which resulted in 64 codes, which were then organized into 12 themes (for an overview of these, see Table 1) that were reviewed to gain an understanding of how the robots affected the human-inhabited real-life work environment.

In this paper, we highlight three key themes in the following section.

Table 1. Overview of themes

Themes identified in the empirical data

\begin{tabular}{cccc}
\hline Reliance and & Attitude & Perceptions & Environment \\
trust & Behaviour & Irritation/frustration & Division of labour \\
Errors & Workarounds & Tempo/pace of work & War stories \\
Conflicts & Hand-overs & Safety & Technique \\
Break downs & & \\
\hline
\end{tabular}

\section{Results}

\subsection{Adjustments}

A primary finding was that the robots had difficulties adjusting to the basement environment and meeting the human expectations of them. The hallways were narrow and not designed for mixed traffic and were therefore divided into two lanes: one for vulnerable users, such as pedestrians and cyclists, and one for heavy users, such as the trucks used by the porters (see Figure 1). It was unclear to the informants in this study whether the robots belonged to the vulnerable or heavy user groups as they were not 
formally assigned to either lane. In collaboration with the robots' developers, the hospital made changes to the hallway junctions, assigning robot lanes with the inscription MiR (the product name of the robot). These mark-ups were designed to inform people in the basement how the robots would make their way through the junctions, and the hospital expected that the robots would remain in their lanes.
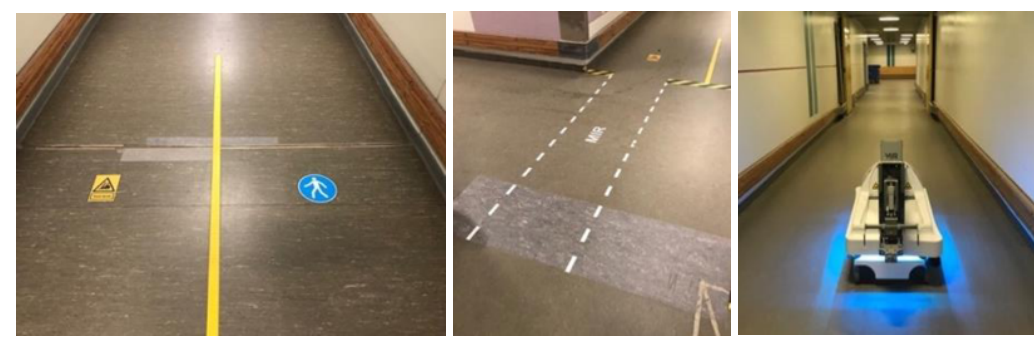

Figure 1. Left: lanes marked for vulnerable and heavy traffic in the hospital cellar hallway. Middle: robot lane marked at a hallway junction. Right: robot driving through a hallway in the hospital basement.

However, this study found that the mobile service robots were unable to adapt to the environmental changes made by humans to ease their routines, such as by failing to drive in the assigned lanes as the humans expected them to. One of the reasons the robots did not use the lanes was that they had to adjust to factors within the hospital basement environment by avoiding porters on trucks, pedestrians, cyclists and items left on the floor. The narrow hallways also brought humans and robots close very close to each other, and if a robot came close to a human, object or item, its sensors would register a potential risk of causing harm, which would immediately make the robot stop what it was doing. These robotic stops would cause frustration and annoyance among the staff, who did not consider that the robots acted as they did in order to avoid harming their surroundings. The humans in the basement were simply unaware that their expectations of seamless, autonomous robot performance were unrealistic in practice.

\subsection{Work-arounds}

A variety of factors, including the parties' mutual impact, affected human-robot cooperation in the hospital basement. As mentioned above, the environment was not suited to the robots, not least because the hallways could get crowded, such as when humans, who had their own daily paths through the hallways, took up space, hindering the robots in performing their tasks. When the mobile robots drove around the humans and other obstacles in the hallways, they would in turn affect the routines of the workers. The robots drove at a slow pace for safety reasons, and the workers in the basement became increasingly irritated and frustrated at sharing their workspace with the robots, not least because they had expected the robots to seamlessly integrate, for example by adjusting to the pace of the hospital staff. Frustrations developed among both kitchen staff (because the robots disturbed their routines and pace of work and thus reduced performance) and by the porters (who already had a tense relationship with the robots, simply by their presence in the basement).

During the field study, it was observed that eight out of the 16 kitchen staff chose to perform themselves tasks that the robots were supposed to do, because they felt they could not rely on the robots. Only by monitoring the robots on a tablet could the staff be sure where the robots were, but not how (or what) they were doing. Further, the robots 
caused the workers' pace to slow, such as when taking up space in the hallway, driving around obstacles and not following lanes (often driving in the middle of the hallway), which made the workers unable to get around them and perform their tasks at the pace they wanted to. The workers had to adjust to the robots because the robots did not adjust to take seamless part in the shared work environment.

\subsection{Sabotage}

It was clear that the porters perceived the robots differently from the kitchen staff. The porters did not approve of the mobile service robots being part of their working environment because they had a large impact on the pace of the porters' working routines as they drove around the hallways at their programmed speed in order to avoid harming their surroundings. The porters also had limited options to get around the robots in the narrow hallways and did not benefit from their presence. Some porters were unwilling to adjust to the robots and would not hesitate to interfere or sabotage them, which resulted in the robots stopping in the performance of their tasks, and it was not uncommon for frustrated workers to turn off the robots, lock them in rooms or pour food into them. The interference and sabotage were a result of the robots affecting the workers' everyday routines. As a consequence, the kitchen staff became frustrated towards both the robots and the porters because they could not rely on the robots to complete their tasks in part due to the porters' actions. The kitchen staff were unable to trust the robots because they knew from experience that errors would eventually occur that would make the robots slow down, abort their missions or simply stop. The kitchen staff could not be sure what type of error (technical or human-triggered) the robots might have encountered, but they repeatedly experienced how the human presence in the basement affected the robots, which they had not expected would be an issue, when the robots were installed. Accordingly, the kitchen staff, who were supposed to benefit from the robots, could not trust them because of the actions of other humans and because they had higher expectations of the robots than the robots were able to meet.

\section{Discussion}

The findings highlight the importance of considering socio-technical factors when deploying robots to cooperate with humans in hospital environments. One of these factors is the human expectations of the robots: the hospital staff expected the mobile robots to be a 'plug-and-play' solution, integrating seamlessly and aiding in the real-life environment. Our research has shown that human expectations of cooperating with robots in the wild may be greater than the robots can meet because of three dominant factors. First, the environmental preparations made by the hospital and the robot developers were ineffectual, yet resulted in raised hopes and great expectations from the hospital staff in the basement. Second, the robots were sensitive to staff behaviour, which caused frustration among the staff resulting in robots being prevented from performing their tasks. Third, the staff could not rely on the robots to aid in everyday work situations.

Hence, the robots did not adjust to the changes made by the developers and the hospital but rather to factors in the environment, such as human behaviour, without the staff realising it. Because of the nature of these adjustments, the robots did not drive in an advantageous, reliable way, as had been expected when they were installed, because the basement environment was not suited for this type of robot. In the given case, these 
factors could not necessarily have been predicted in advance of deployment, as the hospital did not test the robots in a pilot implementation. If the hospital had tested the robots prior to installing them, the factors that were results of the dynamics and interactions between the actors (both humans and robots) and their actions, might have been discovered and suitable adjustments could have been made before the final installation of the robots.

The hospital staff would unintentionally leave items (boxes, containers, or components) in the hallways without reflecting on the consequences, as they were unaware of the robots being sensitive to obstacles and unaware that their behaviour would thus have a large impact on the behaviour of the robots. When the robots encountered obstacles that they were unable to avoid, they would simply stop and wait for the obstacle to disappear. This could lead to a robot not completing its tasks but rather simply standing still while running out of power. The hospital staff who were supposed to benefit from the robots therefore did not rely on them to do what they ought to because of the actions of other humans. The aim of deploying the robots in the hospital kitchen was to ease the burden on kitchen staff to perform logistical tasks in a time-efficient manner, but the assistance was shown to be complex as the kitchen staff found themselves becoming caretakers for the robots, despite the robots being deployed to take care of tasks. The intention of the human-robot cooperation in this hospital was that the robots would support and relieve the hospital kitchen staff, but the hospital kitchen staff instead supported and relieved the robots.

The lack of attention to socio-technical factors in deploying and using robots in this hospital was rooted in techno-optimism, great expectations and the opinions of technology enthusiasts who did not consider how to reorganise existing environments, routines and everyday structures. As argued by Blond et al. [10], the use and meaning of robots are created in practice and cannot be designed in advance. When robots move beyond highly structured environments, the visions and expectations of deploying them in real-life settings must be adapted to those settings. The human expectations of robots cannot be met if they are not considered in relation to the environment in which the robots participate.

Therefore, if robots are to succeed in engaging with real-life environments - rather than reflecting the aspirations of techno-enthusiasts who expect robots to be simple, automatic 'plug-and-play' solutions, without considering the robots' requirements, human adaptations and environmental changes - attention must be paid to the practice in which the robots are to participate, including the socio-technical factors.

\section{Conclusion}

In this paper, we have reported insights from research on how robots affect the real-life environments into which they are gradually moving. The findings showed that the cooperation between humans and robots in the hospital environment was fragmented because of limited attention having been paid to socio-technical factors. Knowledge of real-life environmental factors; human reactions and behaviour towards robots in complex environments not designed for robots; and robotic influences on the environment in which they act has been summarised, and we have identified three major factors that influence human-robot cooperation in hospitals: environmental factors, behavioural factors and factors related to human reliance on robots. 


\section{References}

[1] J. Forlizzi, How robotic products become social products: An ethnographic study of cleaning in the home. HRI 2007 - Proceedings of the 2007 ACM/IEEE Conference on Human-Robot Interaction - Robot as Team Member (2007), 129-136.

[2] J.-Y. Sung, R.E. Grinter, H.I. Christensen, L. Guo, Housewives or technophiles? Understanding domestic robot owners. Proceedings of the $3^{\text {rd }}$ ACM/IEEE Conference on Human-Robot Interaction (2008), 129136.

[3] B. Mutlu, J. Forlizzi, Robots in organizations: The role of workflow, social, and environmental factors in human-robot interaction. Proceedings of the $3^{\text {rd }}$ ACM/IEEE International Conference on Human-Robot Interaction (2008), 287-294.

[4] S. Ljungblad, J. Kotrboca, M. Jacobsen, H. Cramer, K. Niechwiadowicz, Hospital robot at work: Something alien or an intelligent colleague? Proceedings of ACM Conference on Computer Supported Cooperative Work, CSCW (2012), 177-186.

[5] P. Biernacki, D. Waldorf, Snowball sampling: Problems and techniques of chain referral sampling, Sociological Methods \& Research 10, 2 (1981), 141-163.

[6] W. Sperschneider, K. Bagger, Ethnographic fieldwork under industrial constraints: Towards Design-inContext. International Journal of Human Computer Interaction (2003), 41-50.

[7] J.P. Spradley, The ethnographic interview. Holt, Rinehart and Winston, New York, USA, 1979.

[8] M.C. Everett, M.S. Barrett, Guided tour: A method for deepening the relational quality in narrative research. Qualitative Research Journal 12, 1 (2012), 32-46.

[9] V. Braun, V. Clark, Using thematic analysis in psychology, Qualitative Research in Psychology 3, 2 (2006), 77-101.

[10] L. Blond, Studying robots outside the lab: HRI as ethnography, Paladyn, Journal of Behavioral Robotics 10, 1 (2019), 117-127. 